\title{
Author Correction: Molecular basis for acetyl-CoA production by ATP-citrate lyase
}

\author{
Xuepeng Wei ${ }^{(D}$, Kollin Schultz ${ }^{D}$, Gleb A. Bazilevsky, Austin Vogt and Ronen Marmorstein (D)
}

Correction to: Nature Structural \& Molecular Biology https://doi.org/10.1038/s41594-019-0351-6, published online 23 December 2019.

In the version of the article initially published, the phospho-citryl-CoA intermediate in the ACLY-E599Q-ATP-citrate-CoA structure (PDB 6UUW) was modeled incorrectly. Specifically, the citryl-CoA linkage was modeled with a thioether bond instead of a thioester bond, and the phosphate group was connected to the - $\mathrm{OH}$ of the citryl moiety in the phospho-citryl portion rather than via an ester bond with the carboxylate group, as supported by Walsh and Spector (J. Biol. Chem. 243, 446-448, 1968). Thus, the intermediate shown in Fig. 6 and Extended Data Fig. 7c,d was incorrect. The PDB coordinate file has been revised and the original and corrected Fig. 6 and Extended Data Fig. 7c,d are given here. The text has not been altered to reflect these changes, but given the inherent instability of the modeled phospho-citryl-CoA intermediate and the limited resolution of the current structure, the modeled intermediate could represent phospho-citrate + CoA, citryl-CoA + phosphate or a mixture of the two. Given these possibilities, the precise catalytic role of E599 is unknown and E599 may not be directly involved in cleavage of the citryl-CoA adduct, as suggested in the article.

In addition, in Table 1, the number of ligand atoms in the "E599Q with ATP-citrate-CoA (D2)" structure was incorrectly listed as 4; the correct number is 8 . Also in Table 1 , the bond length r.m.s. deviation in the "With citrate-CoA (C1 assym. closed)" structure was incorrectly listed as $0.0008 \AA$; the correct value is $0.008 \AA$. The ACLY-E599Q mutant was incorrectly identified as ACLY-E599A in the following locations: in Results section "ACLY catalysis proceeds through a phospho-citryl-CoA intermediate in the ASH domain", paragraph 16, phrase "We therefore mixed the ACLY-E559Q mutant with saturating concentrations ..."; in the Discussion, paragraph 17, phrase "Interestingly, citrate was added to both the ACLY-citrate-CoA and ACLY-E599Q-ATP-citrate-CoA structures ..."; and in the Discussion, paragraph 20, sentence "This conclusion is further supported by our ability to trap a phospho-citryl-CoA intermediate bound to ACLY-E599Q catalytic mutant." In the Methods section "Cryo-EM data collection, image processing, model building and refinement", paragraph 28, the mutated residue Q was excluded from the name of the mutant in the phrase "For 3D reconstruction of the ACLY-E599Q-citrate-ATP-CoA structure ...." In Extended Data Fig. 4b, incorrect residue ranges were given for helical segments from the CSH domain of ACLY: the residue range was labeled as "resi 1055-1088" in the left and right panels, and the figure caption gave it as "residues 1055-1080." The correct residue range label is "resi 1055-1077" and the correct figure caption text is "Cryo-EM density of representative helical segments (residues 1055-1077) from ACLY-citrate-CoA (left) and ACLY-OAA-acetyl-CoA structures." The errors have been corrected in the HTML and PDF versions of the article.

Published online: 2 April 2020

https://doi.org/10.1038/s41594-020-0421-9

(c) The Author(s), under exclusive licence to Springer Nature America, Inc. 2020 


\section{AMENDMENTS}

NATURE STRUCTURAL \& MOLECULAR BIOLOGY

a

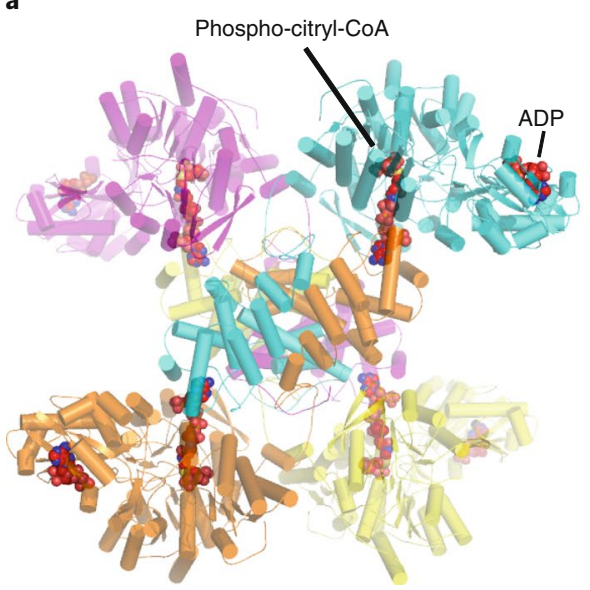

\section{Original}

b
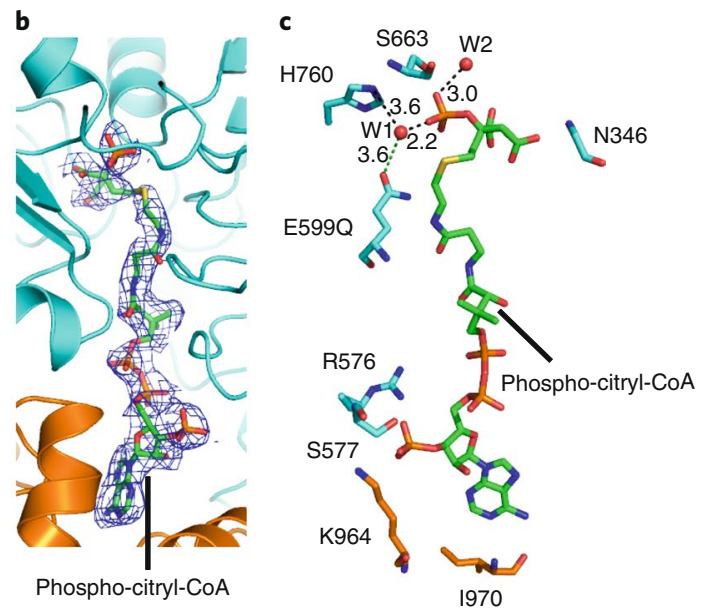

a

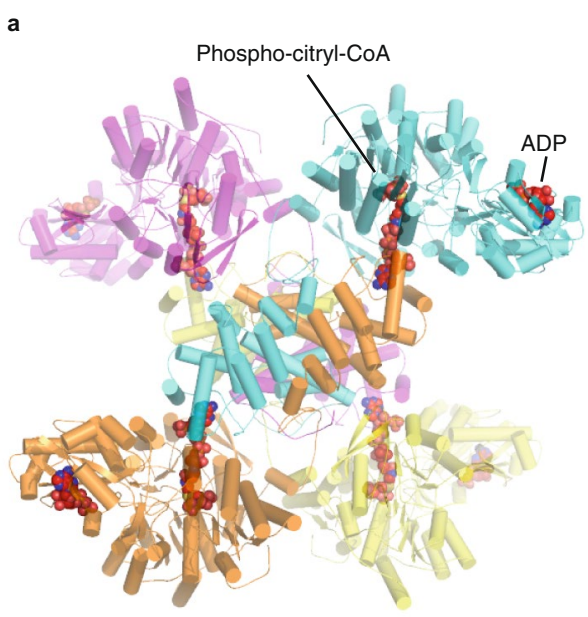

Corrected

b

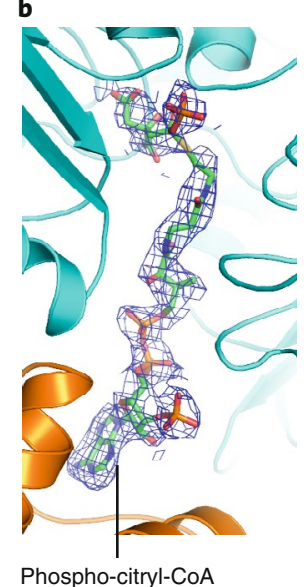

c

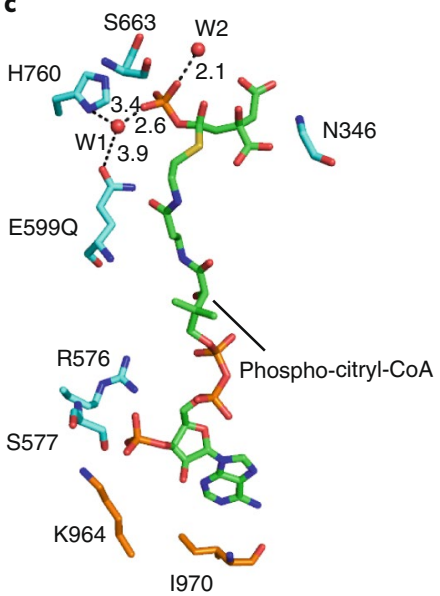

Fig. 1 | Original and corrected Fig. 6. 
c

Original d

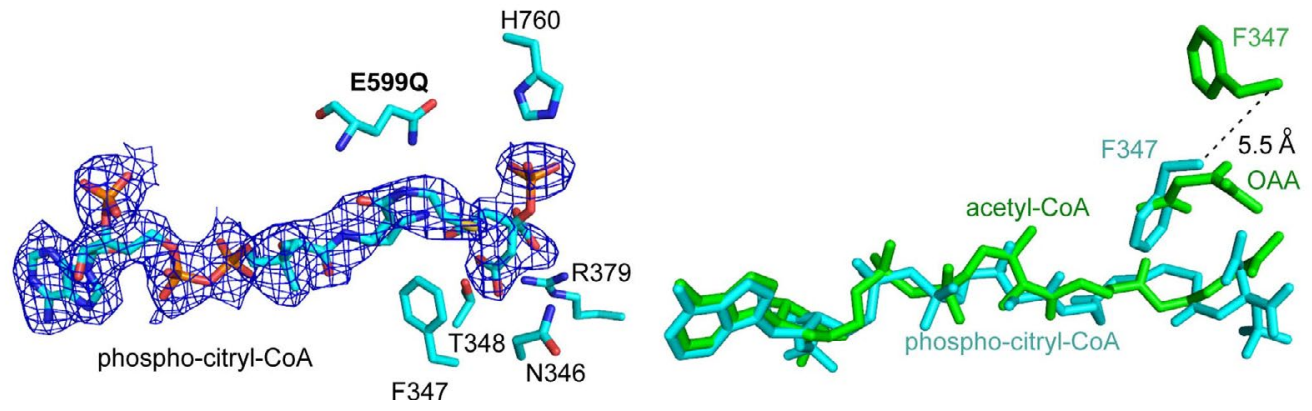

c

Corrected

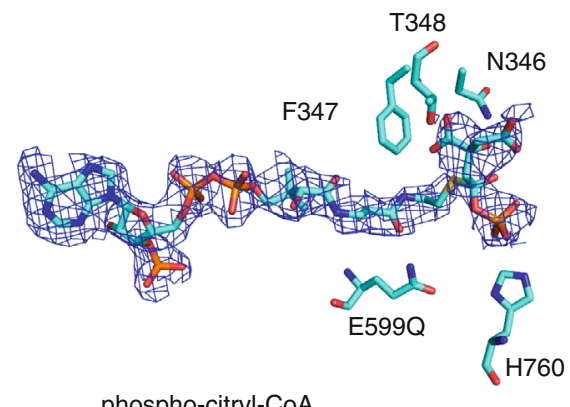

phospho-citryl-CoA d

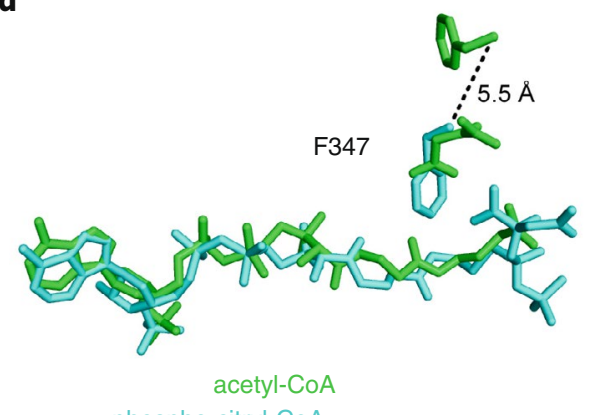

phospho-citryl-CoA

Fig. 2 | Original and corrected Extended Data Fig. 7c,d. 